\title{
Concise total synthesis of cytotoxic natural products (+) and (-)- muricatacin
}

\author{
Srinivasarao Yaragorla and Ramaiah Muthyala* \\ Department of Experimental and Clinical Pharmacology, University of Minnesota, Minneapolis, \\ MN 55455, USA \\ E-mail: muthy003@umn.edu
}

\begin{abstract}
A short and efficient total synthesis of antitumor natural products (+) and (-)-muricatacin is described in four steps with an overall yield of $47 \%$. The key reactions involved in the synthesis are $\mathrm{Ph}_{3} \mathrm{P}$ mediated isomerization and asymmetric dihydroxylation.
\end{abstract}

Keywords: Triphenylphosphine mediated isomerization, natural product, butyrolactone, dihydroxylation, total synthesis, muricatacin

\section{Introduction}

$\gamma$-Butyrolactones (Figure 1) are an important class of natural products with intriguing biological activities ${ }^{1}$ such as antitumor, ${ }^{1 \mathrm{~d}, 1 \mathrm{i}}$ antiparasitic, ${ }^{\mathrm{e}}{ }^{\mathrm{e}}$ antibacterial ${ }^{1 \mathrm{f}}$ and pesticidal. Muricatacin 1, 2, a simple biologically active $\gamma$-butyrolactone derivative, belongs to acetogenin class of natural products and was isolated from seeds of the plant Annona muricata in 1991 by McLaughlin et al., ${ }^{2}$ as a mixture of both enantiomers, with (-) enantiomer as the predominant enantiomer (ca $25 \%$ ). Interestingly, both isomers show potent cytotoxic activity against a variety of human tumor cell lines. ${ }^{2}$ Further, their structure activity relationship studies revealed that the antitumor activities depend mainly upon the length of the alkyl side chains and stereochemistry. ${ }^{3}$ Several groups have published total synthesis of $(+)$ or (-)-muricatacin or both ${ }^{4}$ and their epi-or aza analogues. ${ }^{5}$ Recently Popsavin et al., reported that some of the muricatacin analogues are several fold more potent than the original molecule. ${ }^{6}$ During the course of our ongoing research related to toxic shock syndrome, ${ }^{7}$ we have examined natural products containing long chain alkyl groups with free hydroxyl groups as possible antibacterial agents due to their similarities with glycerol monolaurate. ${ }^{7}$ In continuation of our interest in the synthesis of this class of natural products, herein we report a concise total synthesis of $(+)$ and (-)-muricatacin. This method enables the preparation of a variety of side chain analogues of the natural product for extensive microbiological screening. 
<smiles>[R]CC([R])C1CCC(=O)O1</smiles>

$\mathrm{R}=\mathrm{H}, \gamma$-butyrolactones

$\mathrm{R}=\mathrm{OH}$, hydroxy $-\gamma$-butyrolactones

$\mathrm{R}_{\mathrm{l}}=$ alkyl/aryl

\section{Figure 1}

\section{Results and Discussion}

Our synthetic plan was based on our earlier work, ${ }^{8 \mathrm{e}}$ namely the triphenylphosphine-mediated isomerization of acetylenic esters to the corresponding $(E, E)-\alpha \beta-\gamma \delta$-diene esters, which has attracted considerable attention in the total synthesis of many other natural products. ${ }^{8}$ Sharpless Asymmetric Dihydroxylation (SAD) ${ }^{9}$ on diene ester is employed as the next key reaction to complete the synthesis of (+) and (-)-muricatacin. ${ }^{4 a}$ A similar dihydroxylation on diene ester was reported by O'Doherty et al. ${ }^{4 \mathrm{u}}$ However, the advantage of our method is a two step synthesis of $(E, E)-\alpha \beta$ - $\gamma \delta$-diene ester from the alkyne with improved yields whereas the same diene ester synthesis was achieved in five steps starting from the corresponding alcohol.
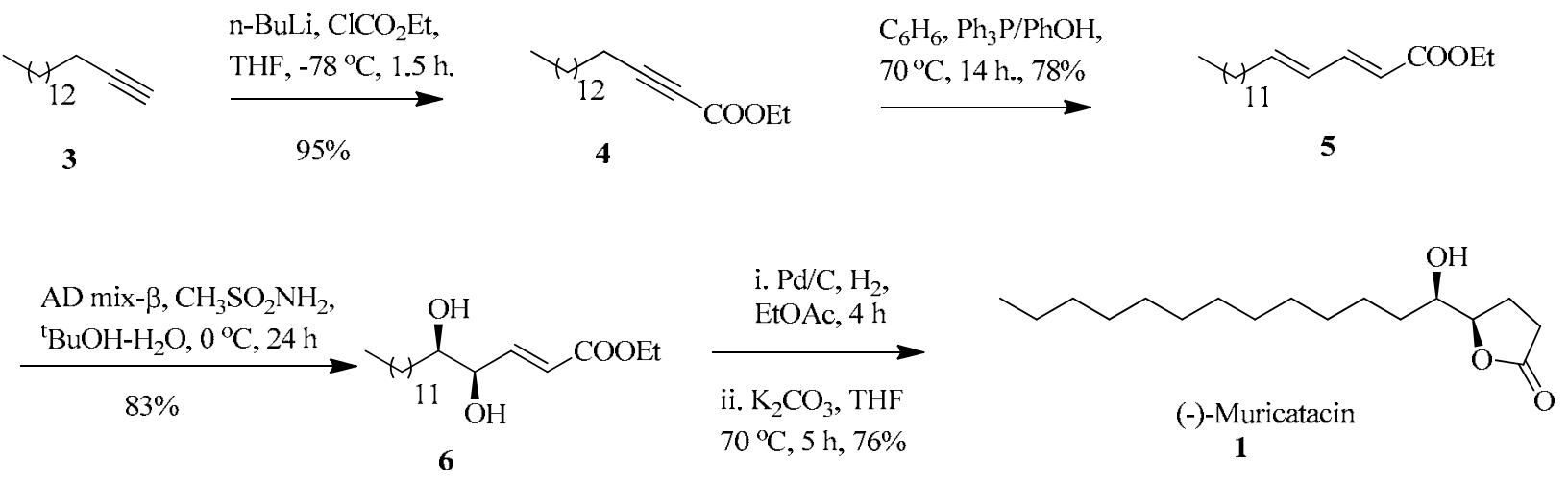

Scheme 1. Synthesis of (-) muricatacin.

As shown in the Scheme 1, the synthesis of both enantiomers of muricatacin commenced with the commercially available 1 -hexadecyne 3 . This was lithiated using $n$-butyllithium at -78 ${ }^{\circ} \mathrm{C}$, and treated with ethyl chloroformate to form the alkyne-ester 4 in $95 \%$ yield. This alkyne 
ester was ready to form the requisite key intermediate (diene ester) 5 for the synthesis of both enantiomers of the target natural product under modified Trost isomerization conditions. ${ }^{8}$ Thus, alkynoate 4, was treated with $\mathrm{Ph}_{3} \mathrm{P} / \mathrm{PhOH}$ in benzene at room temperature for $14 \mathrm{~h}$ to obtain exclusively $(E, E)-\alpha \beta-\gamma \delta$-diene esters 5 in $78 \%$ yield. Enantio- and regio-selective Sharpless dihydroxylation of diene ester 5 with $\mathrm{AD}$ mix $\beta$ - gave $(\gamma \delta)$-dihydroxy, $(\alpha \beta)$-unsaturated esters 6 as a single product in $83 \%$ yield in $24 \mathrm{~h} .{ }^{9}$ Hydrogention of diol 6 with $\mathrm{Pd} / \mathrm{C}$ in ethyl acetate for 4 $\mathrm{h}$, followed by refluxing with $\mathrm{K}_{2} \mathrm{CO}_{3}$ in THF for $5 \mathrm{~h}$ furnished (-)- muricatacin 1 in $76 \%$ yield. In the similar manner (+)-muricatacin 2 was also synthesized from diene ester 5 (Scheme 2), SAD of 5 with AD-mix $\alpha$ furnished diol 7 in $82 \%$ yield which was subjected to $\mathrm{Pd} / \mathrm{C}$ catalyzed hydrogenation (ethyl acetate, $4 \mathrm{~h}$ ) followed by refluxing with $\mathrm{K}_{2} \mathrm{CO}_{3}$ (THF, $5 \mathrm{~h}$ ) gave (+)muricatacin 2 in $76 \%$ overall yield for two steps. The spectral and physical data of our synthetic $(-)$ and $(+)$-muricatacin were in agreement with published literature. ${ }^{4 \mathrm{u}}$

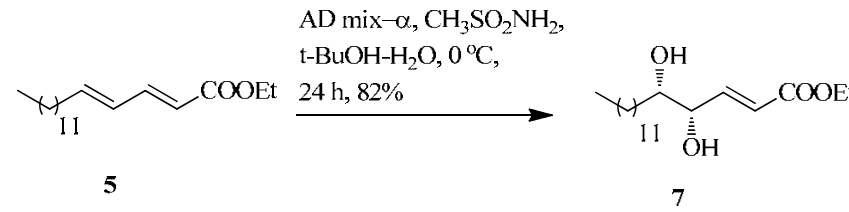

Scheme 2. Synthesis of $(+)$ muricatacin.

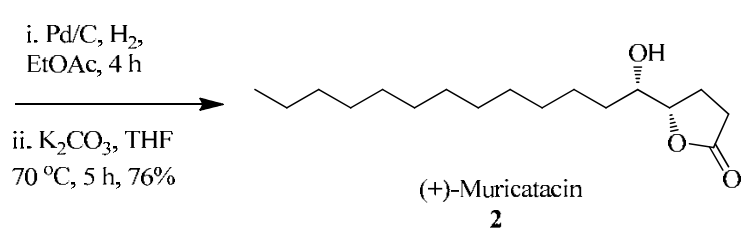

\section{Conclusions}

In conclusion, an efficient total synthesis for both enantiomers of antitumor natural product muricatacin was described in four steps using triphenylphosphine mediated isomerization of alkynoate ester to diene ester and Sharpless asymmetric dihydroxylation as key steps. We expect this method to be useful for the synthesis of a variety of side chain analogues and also applicable for large scale synthesis.

\section{Experimental Section}

General. ${ }^{1} \mathrm{H}$ NMR and ${ }^{13} \mathrm{C}$ NMR spectra were recorded in $\mathrm{CDCl}_{3}$ solvent on BRUKER-400 $\mathrm{MHz}$ spectrometer at ambient temperature. Chemical shifts $\delta$ are given in ppm, coupling constant $J$ are in Hz. The chemical shifts are reported in ppm on scale downfield from TMS as internal standard and signal patterns are indicated as follows: s, singlet; $d$, doublet; $t$, triplet; m, multiplet; br, broad peak. FTIR spectra were recorded as $\mathrm{NaCl}$ thin films. Optical rotations were measured on digital polarimeter (Autopol V) using a $2 \mathrm{~mL}$ cell with a $100 \mathrm{~mm}$ path length. For low (MS) and High (HRMS) resolution, $\mathrm{m} / \mathrm{z}$ ratios are reported as values in atomic mass units (Waters, Micromass). All reagents and solvents were reagent grade and used without further purification 
unless specified otherwise. Column chromatography was carried out using silica gel (230-400 mesh) packed in glass columns. All reactions were performed under an atmosphere of nitrogen in flame-dried or oven-dried glassware with magnetic stirring.

Ethyl heptadec-2-ynoate (4). To a stirred solution of hexadecyne $3(0.8 \mathrm{~g}, 3.6 \mathrm{mmol})$ in dry THF $(10 \mathrm{~mL})$ at $-78{ }^{\circ} \mathrm{C}$ was added $n$-BuLi $(2.25 \mathrm{~mL}, 1.6 \mathrm{M}$ in hexanes, $3.6 \mathrm{mmol})$ slowly under $\mathrm{N}_{2}$ atmosphere and stirred for $15 \mathrm{~min}$ at same temperature. Ethyl chloroformate $(0.28 \mathrm{~mL}, 3.6$ mmol) was added to the reaction mixture and stirring was continued for $1 \mathrm{~h}$ at the same temperature. Saturated $\mathrm{NH}_{4} \mathrm{Cl}(5 \mathrm{~mL})$ was added to the reaction mixture at $-78{ }^{\circ} \mathrm{C}$ and slowly warmed to $\mathrm{rt}$, ethyl acetate $(10 \mathrm{~mL})$ was added to the reaction mixture and organic layer was seperated. The aqueous layer was extracted with EtOAc $(2 \times 10 \mathrm{~mL})$, and the combined organic layers were washed with brine $(10 \mathrm{~mL})$, dried over $\mathrm{Na}_{2} \mathrm{SO}_{4}$ and the solvent was evaporated under reduced pressure. The crude product was purified by silica gel column chromatography using hexanes and ethyl acetate as the eluents (19:1) to get ester 4 as pale yellow oil in 95\% yield. ${ }^{1} \mathrm{H} \mathrm{NMR}\left(\mathrm{CDCl}_{3}, 400 \mathrm{MHz}\right): \delta 4.14(\mathrm{q}, J=14.3,7.1 \mathrm{~Hz}, 2 \mathrm{H}), 2.25(\mathrm{t}, J=7.1 \mathrm{~Hz}, 2 \mathrm{H}), 1.55-1.46$ (m, 2H), 1.37-1.28 (m, 2H), 1.27-1.61 (m, 23H), $0.81(\mathrm{t}, \mathrm{J}=6.6 \mathrm{~Hz}, 3 \mathrm{H}) ;{ }^{13} \mathrm{C} \mathrm{NMR}\left(\mathrm{CDCl}_{3}, 100\right.$ MHz): $\delta 153.8,89.4,73.1,61.7,31.9,29.66,29.63,29.61,29.56,29.4,29.3,29.0,28.8,27.5$, 22.6, 18.6, 14.1, 14.0; IR (film) $v_{\max }: 2925,2854,2235,1714,1465,1366,1249,1074 \mathrm{~cm}^{-1}$; Mass (ESI, $m / z): 295.4(\mathrm{M}+\mathrm{H})^{+}$.

Ethyl (2E,4E)-heptadeca-2,4-dienoate (5). A mixture of ester $4(1.0 \mathrm{~g}, 3.4 \mathrm{mmol})$ and Triphenylphosphine $(1.34 \mathrm{~g}, 5.1 \mathrm{mmol})$ was refluxed in benzene $(10 \mathrm{~mL})$ with a catalytic phenol $(0.5 \mathrm{~mL})$ for $14 \mathrm{~h}$. After completion of the reaction (monitored by TLC), the solvent was removed under vacuum and the crude product was purified on a silica gel column (eluted with $4 \%$ ethyl acetate in hexanes), furnished (E, E)- $\alpha \beta-\gamma \delta$-unsaturated ester 5 as a colorless oil in $78 \%$ yield.

${ }^{1} \mathrm{H}$ NMR $\left(\mathrm{CDCl}_{3}, 400 \mathrm{MHz}\right): \delta$ 7.21-7.15 (m, 1H), 6.13-6.00 (m, 2H), $5.70(\mathrm{~d}, J=15.4 \mathrm{~Hz}, 1 \mathrm{H})$, $4.03(\mathrm{q}, J=7.1,2.0 \mathrm{~Hz}, 2 \mathrm{H}), 2.08(\mathrm{q}, J=13.2,6.7 \mathrm{~Hz}, 2 \mathrm{H}), 1.62-1.53(\mathrm{~m}, 1 \mathrm{H}), 1.39-1.28(\mathrm{~m}$, $3 \mathrm{H}), 1.27-1.15(\mathrm{~m}, 19 \mathrm{H}), 0.86(\mathrm{t}, J=7.4 \mathrm{~Hz}, 3 \mathrm{H}) ;{ }^{13} \mathrm{C} \mathrm{NMR}\left(\mathrm{CDCl}_{3}, 100 \mathrm{MHz}\right): \delta 167.3,145.1$, 144.7, 128.3, 119.1, 60.1, 33.0, 31.9, 30.7, 29.6, 29.58, 29.4, 29.3, 29.2, 28.7, 22.6, 14.3, 14.1; IR (film) $v_{\max }: 2925,2854,1725,1660,1642,1466,1302,1265,1178 \mathrm{~cm}^{-1}$; Mass (ESI, m/z): 295.2 $(\mathrm{M}+\mathrm{H})^{+}$; HRMS (ESI, $\left.m / z\right)$ : calculated for $\mathrm{C}_{19} \mathrm{H}_{35} \mathrm{O}_{2}(\mathrm{M}+\mathrm{H})^{+} 295.2481$, found -295.2479 .

Ethyl (4S,5S,E)-4,5-dihydroxyheptadec-2-enoate (6). ADmix- $\beta \mathrm{K}_{2} \mathrm{OsO}_{2}(\mathrm{OH})_{4}+\mathrm{K}_{3} \mathrm{Fe}(\mathrm{CN})_{6}+$ $\mathrm{K}_{2} \mathrm{CO}_{3}+\left((\mathrm{DHQD})_{2} \mathrm{PHAL}\right]$ (4.76 g, $1.4 \mathrm{~g}$ for $1 \mathrm{mmol}$ of olefin) was dissolved in $t$-BuOH $(5 \mathrm{~mL})$ and $\mathrm{H}_{2} \mathrm{O}(5 \mathrm{~mL})$. Methanesulfonamide $(325 \mathrm{mg}, 3.4 \mathrm{mmol})$ and diene $5(1.0 \mathrm{~g}, 3.4 \mathrm{mmol})$ were then added at $0{ }^{\circ} \mathrm{C}$ and the reaction vigorously stirred for $24 \mathrm{~h}$ at same temperature. After complete consumption of the starting material, $\mathrm{Na}_{2} \mathrm{SO}_{3}(1 \mathrm{~g})$ was then added and the solution stirred for $1 \mathrm{~h}$ after which the reaction was poured into water $(10 \mathrm{~mL})$ and extracted with EtOAc $(3 \times 10 \mathrm{~mL})$. The combined organics were washed with brine $(10 \mathrm{~mL})$, and dried over anhydrous $\mathrm{Na}_{2} \mathrm{SO}_{4}$. The solvent was removed under reduced pressure to yield the crude diol which was 
purified by silica gel column chromatography using EtOAc/hexanes (1:4) to yield pure diol 6 in $83 \%$ yield as a white solid.

${ }^{1} \mathrm{H} \mathrm{NMR}\left(\mathrm{CDCl}_{3}, 400 \mathrm{MHz}\right): \delta 6.93(\mathrm{dd}, J=15.6,5.0 \mathrm{~Hz}, 1 \mathrm{H}), 6.13(\mathrm{dd}, J=15.6,1.5 \mathrm{~Hz}, 1 \mathrm{H})$, 4.20 (q, $J=14.3,7.1 \mathrm{~Hz}, 2 \mathrm{H}), 4.12$ (t, $J=4.5 \mathrm{~Hz}, 1 \mathrm{H}), 3.57-3.53(\mathrm{~m}, 1 \mathrm{H}), 2.59(\mathrm{~s}, 1 \mathrm{H}), 2.20(\mathrm{~s}$, $1 \mathrm{H}), 1.59-1.41(\mathrm{~m}, 3 \mathrm{H}), 1.40-1.17(\mathrm{~m}, 22 \mathrm{H}), 0.87(\mathrm{t}, J=6.6 \mathrm{~Hz}, 3 \mathrm{H}) ;{ }^{13} \mathrm{C} \mathrm{NMR}\left(\mathrm{CDCl}_{3}, 100\right.$ $\mathrm{MHz}): \delta 166.4,146.9,122.5,74.1,74.0,60.6,33.1,31.9,29.7,29.66,29.61,29.59,29.3,25.6$, 22.7, 14.2, 14.1; IR (film) $v_{\max }: 3277,2955,2917,2846,1708,1665,1464,1276 \mathrm{~cm}^{-1}$; Mass (ESI, m/z): $329.2(\mathrm{M}+\mathrm{H})^{+}$; HRMS (ESI, m/z): calculated for $\mathrm{C}_{19} \mathrm{H}_{37} \mathrm{O}_{4}(\mathrm{M}+\mathrm{H})^{+} 329.2535$, found 329.2521; white solid, mp 77-79 ${ }^{\circ} \mathrm{C}$.

Ethyl (4R,5R,E)-4,5-dihydroxyheptadec-2-enoate (7). ${ }^{1} \mathrm{H} \mathrm{NMR}\left(\mathrm{CDCl}_{3}, 400 \mathrm{MHz}\right): \delta 6.93$ $(\mathrm{dd}, J=15.6,5.0 \mathrm{~Hz}, 1 \mathrm{H}), 6.13(\mathrm{dd}, J=15.6,1.6 \mathrm{~Hz}, 1 \mathrm{H}), 4.20(\mathrm{q}, J=14.3,7.1 \mathrm{~Hz}, 2 \mathrm{H}), 4.10$ (t, $J=7.1 \mathrm{~Hz}, 1 \mathrm{H}), 3.57-3.53(\mathrm{~m}, 1 \mathrm{H}), 2.59(\mathrm{~s}, 1 \mathrm{H}), 2.20(\mathrm{~s}, 1 \mathrm{H}), 1.59-1.41(\mathrm{~m}, 3 \mathrm{H}), 1.40-1.17(\mathrm{~m}$, 22H), $0.87(\mathrm{t}, J=6.6 \mathrm{~Hz}, 3 \mathrm{H}) ;{ }^{13} \mathrm{C} \mathrm{NMR}\left(\mathrm{CDCl}_{3}, 100 \mathrm{MHz}\right): \delta 166.2,146.7,122.5,74.1,74.0$, 60.6, 33.1, 31.9, 29.7, 29.66, 29.61, 29.59, 29.3, 25.6, 22.7, 14.2, 14.1; IR (film) $v_{\max }: 3277$, 2955, 2917, 2846, 1708, 1665, 1464, $1276 \mathrm{~cm}^{-1}$; Mass (ESI, m/z): $329.2(\mathrm{M}+\mathrm{H})^{+}$; HRMS (ESI, $\mathrm{m} / \mathrm{z}$ ): calculated for $\mathrm{C}_{19} \mathrm{H}_{37} \mathrm{O}_{4}(\mathrm{M}+\mathrm{H})^{+} 329.2535$, found 329.2519 ; white solid, mp 77-79 ${ }^{\circ} \mathrm{C}$.

(R)-5-((R)-1-Hydroxytridecyl)dihydrofuran-2(3H)-one $\{(-)$-muricatacin $\}(\mathbf{1}):^{4}[\alpha]_{\mathrm{D}}{ }^{20}=-19.1$ (c 1.0, $\left.\mathrm{CHCl}_{3}\right) ;{ }^{1} \mathrm{H} \mathrm{NMR}\left(\mathrm{CDCl}_{3}, 400 \mathrm{MHz}\right): \delta 4.40(\mathrm{dt}, J=7.6,4.8 \mathrm{~Hz}, 1 \mathrm{H}), 3.59-3.51(\mathrm{~m}, 1 \mathrm{H})$, 2.65-2.47 (m, 2H), 2.28-2.18 (m, 1H), 2.18-2.07 (m, 1H), 1.89-1.87 (m, 1H), 1.62-1.49 (m, 3H), 1.45-1.19 (m, 19H), $0.86(\mathrm{t}, J=6.8 \mathrm{~Hz}, 3 \mathrm{H}) ;{ }^{13} \mathrm{C} \mathrm{NMR}\left(\mathrm{CDCl}_{3}, 100 \mathrm{MHz}\right): \delta 177.1,82.9,73.6$, $32.9,31.9,29.61,29.60,29.5,29.4,29.3,28.7,25.5,24.1,22.6,14.1$; IR (neat) $v_{\max }: 3400,2955$, 2918, 2849, 1742, 1472, 1453, $1190 \mathrm{~cm}^{-1}$; Mass (ESI, m/z): $285.2(\mathrm{M}+\mathrm{H})^{+}$. HRMS (ESI, m/z): calculated for $\mathrm{C}_{17} \mathrm{H}_{32} \mathrm{O}_{3}(\mathrm{M}+\mathrm{H})^{+} 285.2430$, found 285.2434; white solid, mp $68-70{ }^{\circ} \mathrm{C}$.

(S)-5-((S)-1-Hydroxytridecyl)dihydrofuran-2(3H)-one $\{(+)$-muricatacin $\}(2):{ }^{4}[\alpha]_{\mathrm{D}}{ }^{20}=+19.6$ $\left.(c) 1.0, \mathrm{CHCl}_{3}\right) ;{ }^{1} \mathrm{H} \mathrm{NMR}\left(\mathrm{CDCl}_{3}, 400 \mathrm{MHz}\right): \delta 4.40(\mathrm{dt}, J=7.6,4.8 \mathrm{~Hz}, 1 \mathrm{H}), 3.59-3.51(\mathrm{~m}, 1 \mathrm{H})$, 2.65-2.47 (m, 2H), 2.28-2.18 (m, 1H), 2.18-2.07 (m, 1H), 1.89-1.87 (m, 1H), 1.62-1.49 (m, 3H), 1.45-1.19 (m, 19H), $0.86(\mathrm{t}, J=6.8 \mathrm{~Hz}, 3 \mathrm{H}) ;{ }^{13} \mathrm{C} \mathrm{NMR}\left(\mathrm{CDCl}_{3}, 100 \mathrm{MHz}\right): \delta 177.1,82.9,73.6$, $32.9,31.9,29.61,29.60,29.5,29.4,29.3,28.7,25.5,24.1,22.6,14.1$; IR (neat) $v_{\max }: 3400,2955$, 2918, 2849, 1742, 1472, 1453, $1190 \mathrm{~cm}^{-1}$; Mass (ESI, m/z): $285.2(\mathrm{M}+\mathrm{H})^{+}$. HRMS (ESI, m/z): calculated for $\mathrm{C}_{17} \mathrm{H}_{33} \mathrm{O}_{3}(\mathrm{M}+\mathrm{H})^{+} 285.2430$, found 285.2427 ; white solid, mp $68-70{ }^{\circ} \mathrm{C}$.

\section{Acknowledgements}

We thank the Institute for Therapeutics Discovery and Development at the University of Minnesota for providing NMR and MS data. The Bob Alison Ataxia Research Center is thanked for their financial support. 


\section{References and Notes}

1. (a) Devon, T. K.; Scott, A. I. In Handbook of Naturally Occuring Compounds, Vol.I, Academic Press, New York, 1975. (b) Mori, K. Tetrahedron 1989, 45, 3233. (c) Friesen, R. W.; Vanderwal, C. J. Org. Chem. 1996, 61, 9103. (d) Alali, F. Q.; Rogers, L.; Zhang, Y.; McLanghlin, J. L. Tetrahedron 1998, 54, 5833. (e) Kotsuki, H.; Miyazaki, A.; Ochi, M. Tetrahedron Lett. 1991, 32, 4503. (f) Koch, S. S. C.; Chambcrlin, A. R. J. Org. Chem. 1993, 58, 2725 and references therein. (g) Buisson, D.; Azerad, R. Tetrahedron: Asymmetry 1996, 7, 9. (h) Pearson, W. H.; Hembre, E. J. J. Org. Chem. 1996, 61, 7217. (i) Warmerdam, E.; Tranoy, I.; Renoux, B.; Gesson, J. -P. Tetrahedron Lett. 1998, 39, 8077.

2. Reiser, M. J.; Kozlowski, J. F.; Wood, K. V.; McLaughlin, J. L. Tetrahedron Lett. 1991, 32, 1137.

3. Cave, A.; Chaboche, C.; Figadère, B.; Harmange, J. C.; Laurens, A. A.; Peyrat, J. F.; Pichon, M.; Szlosek, M.; Cotte- Laffite, J.; Quero, A. M. Eur. J. Med. Chem. 1997, 617.

4. (a) Wang, Z.-M.; Zhang, X.-L.; Sharpless, K. B.; Sinha Subhash, C.; Sinha-Bagchi, A.; Keinan, E. Tetrahedron Lett. 1992, 33, 6407. (b) Marshall, J. A.; Welmaker, G. S. Synlett 1992, 537. (c) Tochtermann, W.; Scholz, G.; Bunte, G.; Wolff, C.; Peters, E.-M.; Peters, K.; Von Schnering, H. G. Liebigs Ann. Chem. 1992, 1069. (d) Marshall, J. A.; Welmaker, G. S. J. Org. Chem. 1994, 59, 4122. (e) Quayle, P.; Rahman, S.; Herbert, J. Tetrahedron Lett. 1995, 36, 8087. (f) Saniere, M.; Charvet, I.; Le Merrer, Y.; Depezay, J. C. Tetrahedron 1995, 51, 1653. (g) Van Aar, M. P. M.; Thijs, L.; Zwanenburg, B. Tetrahedron 1995, 51, 11223. (h) Somfai, P. J. Chem. Soc., Perkin Trans. 1 1995, 817. (i) Bonini, C.; Federici, C.; Rossi, L.; Righi, G. J. Org. Chem. 1995, 60, 4803. (j) Rassu, G.; Pinna, L.; Spanu, P.; Zanardi, F.; Battistini, L.; Casiraghi, G. J. Org. Chem. 1997, 62, 4513. (k) Chang, S.-W.; Hung, C.-Y.; Liu, H.-H.; Uang, B.-J. Tetrahedron: Asymmetry 1998, 9, 521. (1) Szlosek, M.; Franck, X.; Figadere, B.; Cave, A. J. Org. Chem. 1998, 63, 5169. (m) Couladouros, E. A.; Mihou, A. P. Tetrahedron Lett. 1999, 40, 4861. (n) Baylon, C.; Prestat, G.; Heck, M. P.; Mioskowski, C. Tetrahedron Lett. 2000, 41, 3833. (o) Chandrasekhar, M.; Chandra, K. L.; Singh, V. K. Tetrahedron Lett. 2002, 43, 2773. (p) Carda, M.; Rodrı' guez, S.; Gonzalez, F.; Castillo, E.; Villanueva, A.; Marco, J. A. Eur. J. Org. Chem. 2002, 2649. (q) Popsavin, V.; Krstic, I.; Popsavin, M. Tetrahedron Lett. 2003, 44, 8897. (r) Sadagopan, R.; Joseph, S. C. Tetrahedron: Asymmetry 2003, 14, 101. (s) Yoshimitsu, T.; Makino, T.; Nagoaka, H. J. Org. Chem. 2003, 68, 7548. (t) Quinn, K. J.; Isaacs, A. K.; Arvary, R. A. Org. Lett. 2004, 6, 4143. (u) Ahmed, M. M.; Cui, H.; O’Doherty, G. A. J. Org. Chem. 2006, 71, 6686. (v) Prasad, K. R.; Anbarasan, P. Tetrahedron:Asymmetry 2006, 17, 2465. (w). Laurent, F.; Sébastien, R.; Patrice, C.; Cossy, J. Synlett 2007, 2891. (x) Prasad, K, R.; Gandi, V. Tetrahedron: Asymmetry 2008, 19, 2616. (y) Barros, M. T.; Charmier, M. A. J.; Maycock, C. D.; Michaud, 
T. Tetrahedron 2009, 65, 396. (z) Ghosal, P.; Kumar, V.; Shaw, A. K. Carbohydr. Res. 2010, 345, 41.

5. (a) Baussanne, I.; Schwardt, O.; Royer, J.; Pichon, M.; Figadere, B.; Cave, A. Tetrahedron Lett. 1997, 38, 2259. (b) Gypser, A.; Peterek, M.; Scharf, H.-D. J. Chem. Soc., Perkin Trans. 1 1997, 1013. (c) Tsai, S.-H.; Hsieh, P.-C.; Wei, L.-L.; Chiu, H.-F.; Wu, Y.-C.; Wu, M.-J. Tetrahedron Lett. 1999, 40, 1975.

6. Popsavin, V.; Sreco, B.; Benedekovic, G.; Popsavin, M.; Francuz, J.; Kojic, V.; Bogdanovic, G. Bio Org. Med. Chem. Lett. 2008, 18, 5182.

7. Lin, Y. -C.; Schlievert, P. M.; Anderson, M. J.; Fair, C. L.; Schaefers, M. M.; Muthyala, R.; Peterson, M. L. PLoS ONE, 2009, 4, e7499.

8. (a) Rychnovsky, S. D.; Kim, J. J. Org. Chem. 1994, 59, 2659. (b) Trost, B. M.; Kazmaier, U. J. Am. Chem. Soc. 1992, 114, 7933. (c) Guo, C.; Lu, X; J. Chem. Soc., Chem. Commun. 1993, 394. (d) Chandrasekhar, S.; Sultana, S. S. Tetrahedron Lett. 2006, 47, 7255. (e) Yaragorla, S.; Muthyala, R. Tetrahedron Lett. 2010, 51, 467. (f) Reddy, Ch, R.; Rao, N, N. Tetrahedron Lett. 2009, 50, 2478. (g) Chandrasekhar, S.; Parida, B. B.; Rambabu, C. Tetrahedron Lett. 2009, 50, 3294. (h) Chandrasekhar, S.; Vijeender, K.; Chandrasekhar, G.; Reddy, C. R. Tetrahedron: Asymmetry 2007, 18, 2473. (i) Reddy, C. R., Dharmapuri, G.; Rao, N. N. Org. Lett. 2009, 11, 5730.

9. (a) Xu, D.; Crispino, G. A.; Sharpless, K. B. J. Am. Chem. Soc. 1992, 114, 7570. (b) Zhang, Y.; O’Doherty, G. A. Tetrahedron 2005, 61, 6337. 